P220 ROLE OF EBUS-TBNA IN THE DIAGNOSIS OF PRIMARY AND RELAPSING HAEMATOLOGICAL MALIGNANCY

${ }^{1} \mathrm{RM}$ Thakrar, ${ }^{2} \mathrm{G}$ Hardavella, ${ }^{2} \mathrm{JM}$ Brown, ${ }^{2} \mathrm{~L}$ Succony, ${ }^{2} \mathrm{M}$ Falzon, ${ }^{2} \mathrm{E}$ Borg, ${ }^{3} \mathrm{~V}$ Jeebun, ${ }^{4} \mathrm{M}$ Munnavar, ${ }^{2} \mathrm{SM}$ Janes, ${ }^{2} \mathrm{~N}$ Navani. ${ }^{1}$ Lungs for Living Research Centre, University College London, UK; ${ }^{2}$ University College London Hospital, London, UK; ${ }^{3}$ North Tees and Hartlepool NHS Foundation Trust, Stockton-on-Tees, UK; ${ }^{4}$ Lancashire Teaching Hospital, Lancashire, UK

\subsection{6/thoraxjnl-2014-206260.349}

Introduction Endobronchial ultrasound and transbronchial needle aspiration (EBUS-TBNA) has been embraced as a breakthrough in the diagnosis and mediastinal staging of lung cancer. However, its utility in determining a diagnosis of lymphoma is not well defined, and is currently not recommended by the British Thoracic Society.

Aim Evaluate the role of EBUS-TBNA in the diagnosis and subtyping of haematological malignancies.

Method Patients referred with mediastinal lymphadenopathy for EBUS-TBNA in whom lymphoma was suspected, were identified retrospectively in 3 tertiary centres in the UK between 2008 and 2013. EBUS was performed using a linear array ultrasonic bronchoscope and specimens taken with a 21 or 22 gauge needle. The diagnostic accuracy, avoidance of mediastinoscopy and surgical biopsy in cases of primary and relapsing haematological malignancy was recorded. Where EBUS-TBNA was negative, patients subsequently underwent surgical biopsy or clinical and radiological surveillance.

Results Twenty-four patients (10 female and 14 male) with a mean age of 55.5 years underwent EBUS-TBNA for evaluation of mediastinal lymphadenopathy. Clinical and radiological diagnosis was of either, isolated mediastinal lymphadenopathy of unknown cause $(n=15)$ or suspected mediastinal recurrence of lymphoma $(\mathrm{n}=8)$. Five patients $(62.5 \%)$ were found to have a relapse of their haematological malignancy, whilst a new diagnosis of lymphoma was made in 11 cases $(73.3 \%)$ where the presentation was of isolated mediastinal lymphadenopathy. One patient in this group was found to have tuberculosis. Tissue obtained from nodal aspiration was sufficient to subtype the disease in detail in 14 patients with immunohistochemistry. Overall, 17 patients $(70 \%)$ were prevented from having mediastinoscopy and other biopsies, and the diagnostic accuracy and sensitivity of EBUS-TBNA in primary and relapsing lymphoma was 74\%.

Conclusion EBUS-TBNA is a safe and important approach to mediastinal lymphadenopathy in suspected lymphoma, whereby detection of either primary or relapsing haematological malignancy prevents need for other more invasive biopsies.

\section{P221 THE USE OF CYTOLOGICAL SPECIMENS TO DETERMINE EPIDERMAL GROWTH FACTOR RECEPTOR (EGFR) MUTATION STATUS IN NON-SMALL CELL LUNG CANCERS (NSCLC)}

${ }^{1} \mathrm{GH}$ Jones, ${ }^{1} \mathrm{FJ}$ Frost, ${ }^{1} \mathrm{~A}$ Lakhanpal, ${ }^{2} \mathrm{C}$ Smyth, ${ }^{1} \mathrm{M}$ Ledson, ${ }^{1} \mathrm{MJ}$ Walshaw. ${ }^{1}$ Liverpool Heart and Chest Hospital, Liverpool, UK; ${ }^{2}$ Royal Liverpool University Hospital, Liverpool, UK

\subsection{6/thoraxjnl-2014-206260.350}

Introduction Treatments for patients with advanced NSCLC are improving, including the use of drugs which act on those cancers that express EGFR mutations. However, determination of EGFR status requires an adequate sample for analysis and national guidelines indicate that this is best obtained by tissue biopsy. Unfortunately, in this ill patient group tissue biopsy may be problematic and a diagnosis is often made through cytology alone. There are few published studies on the utility of cytological specimens for EGFR analysis and we wished to study this further.

Methods We reviewed all lung cancer samples that had been sent from our large cancer unit since EGFR mutation analysis became available 35 months ago, comparing the yield from cytology and formal tissue biopsy.

Results Of 330 cases sent for EGFR analysis, cytology was the only sample available in 92 cases [28\%] - as might be expected this group contained individuals with poor lung function and high rates of metastatic disease (Mean $\mathrm{FEV}_{1} 0.76 \mathrm{~L}$, Median stage $=4)$. Samples were most commonly obtained from EBUS [59\%], bronchial brushings/washings [23\%], or pleural aspiration [11\%], and 64 [70\%] of these were deemed adequate for mutation analysis.

Although formal histological samples were more likely to provide sufficient material for EGFR analysis than cytological methods [ $84 \%$ vs. $70 \%]$, those acquired through EBUS or pleural aspiration gave comparable rates [ $81 \%$ and $88 \%$ respectively].

Overall, 34 cases [10\%] were EGFR positive, and 4 of these [12\%] were based on cytology samples alone.

Conclusions Our data show that most cytopathological specimens, especially when obtained by EBUS or pleural aspiration, will provide adequate material for EGFR analysis and increase the identification of patients eligible to receive targeted therapy. As cytology specimens can often be obtained through relatively non-invasive means our findings further underline the importance of attempting a tissue diagnosis even in patients who may otherwise be regarded as too unwell for more intensive investigations.

\section{P222 SHORT AND LONG-TERM CONSEQUENCES OF PNEUMOTHORAX FOLLOWING CT-GUIDED LUNG BIOPSY FOR LUNG MALIGNANCY}

E Johnson, A MacKenzie, S Tsim, KG Blyth. Department of Respiratory Medicine, Southern General Hosptial, Glasgow, UK

\subsection{6/thoraxjnl-2014-206260.351}

Introduction and objectives Pneumothorax (PTX) is a common complication of Computed Tomography-guided lung biopsy (CTLB). We sought to quantify the short and long-term consequences of post-CTLB PTX in patients with lung malignancy.

Methods We retrospectively reviewed case records and imaging of all who underwent CTLB in NHS Greater Glasgow and Clyde between August 2011 and August 2012. Patient characteristics (including diagnosis, lung function and blood results), biopsy characteristics, 30-day mortality, PTX management and survival were recorded. Lesion size, depth and maximum PTX depth were directly measured on digitally-stored PACS images.

Patients were classified into no PTX, non-significant PTX (nsPTX) and significant PTX (sPTX), which was defined as any PTX which required pleural intervention. Differences in patient and biopsy characteristics between groups were identified by ANOVA and quantified by Tukey's Multiple Comparisons Test. Kaplan-Meier curves were plotted and differences quantified by log-rank, log-rank for trend and Hazard Ratios. Results are mean $( \pm \mathrm{SD})$.

Results 324 CTLB were performed. Malignancy was confirmed in $285 / 324(88 \%)$. Mean age was $70( \pm 11)$ years. $84 / 285(29 \%)$ developed a PTX. 17/285 (6\%) developed a sPTX. There were 